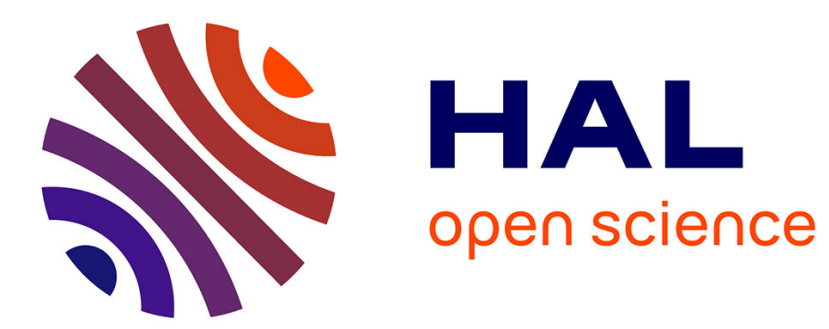

\title{
INCLUSIVE HYPERON POLARIZATION : A REVI EW
}

K. Heller

\section{To cite this version:}

K. Heller. INCLUSIVE HYPERON POLARIZATION : A REVI EW. Journal de Physique Colloques, 1985, 46 (C2), pp.C2-121-C2-129. 10.1051/jphyscol:1985212 . jpa-00224523

\section{HAL Id: jpa-00224523 https://hal.science/jpa-00224523}

Submitted on 1 Jan 1985

HAL is a multi-disciplinary open access archive for the deposit and dissemination of scientific research documents, whether they are published or not. The documents may come from teaching and research institutions in France or abroad, or from public or private research centers.
L'archive ouverte pluridisciplinaire HAL, est destinée au dépôt et à la diffusion de documents scientifiques de niveau recherche, publiés ou non, émanant des établissements d'enseignement et de recherche français ou étrangers, des laboratoires publics ou privés. 
JOURNAL DE PHYSIQUE

Colloque C2, supplément au $n^{\circ} 2$, Tome 46, février 1985

page C2-121

\section{INCLUSIVE HYPERON POLARIZATION : A REVIEW}

K. Heller

School of Physics and Astronomy, University of Minnesota, Minneapolis, Minnesota 55455, U.S.A.

RESUME - On étudie la polarisation des hypérons produits dans la fragmentation des protons pour déterminer la dépendance par rapport aux différents hypérons, à l'énergie du faisceau, à la cible et à la région cinématique considérée. On donne aussi les résultats de la polarisation avec des faisceaux de mésons et de neutrinos.

ABSTRACT . Polarizations from proton fragmentation are compared to determine the dependence on produced hyperon according to energy, target and kinematics. Polarization data for meson and neutrino beams are also given. 
There are many reasons for studying hyperon production. Hyperons after all are the most common of the "stable" baryons comprising $3 / 4$ of the baryon octet. In addition they offer an excellent method of probing the mechanisms of particle production by using the strange quark as a tracer. Spin properties can be measured as easily as cross sections since decay via the parity violating weak interaction automatically reveals the polarization. It has been known for almost ten years that $\Lambda$ hyperons produced by high energy protons are strongly polarized. During the past decade many experiments have been carried out to determine the energy dependence of this polarization, its kinematic behavior, and the extent to which other hyperons are polarized. This work, ${ }_{1}$ summarized in Fig. 1, has shown that the polarization is both large and pervasive.

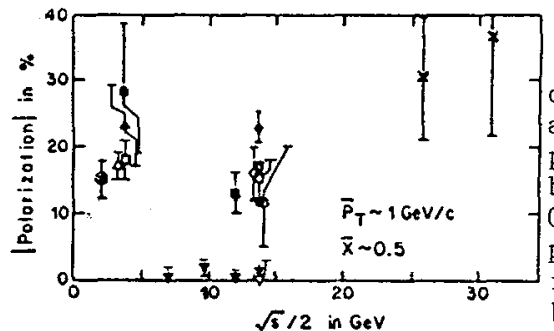

All of the high energy hyperon polarization data comes from inclusive reactions, $\mathrm{A}+\mathrm{B} \rightarrow \mathrm{H}+$ anything, where $A$ is the projectile (usually a proton but there is some data from $\pi, K, \nu$ and $\gamma$ beams), $\mathrm{B}$ is the target (most commonly $\mathrm{Be}$ but $\mathrm{H}_{2}, \mathrm{D}_{2}$, $\mathrm{C}$, $\mathrm{Ir}, \mathrm{Pb}$ and $\mathrm{W}$ have also been used), and $\mathrm{H}$ is the produced hyperon $\left(\Lambda, \Sigma^{+}, \Sigma^{-}, \Sigma^{0}, \equiv^{0}, \equiv^{-}, \Omega^{-}, \bar{\Lambda}\right)$. The fraction of the parent particle momentum retained $\sqrt{5} / 2$ in Gev by the hyperon is $\mathrm{x}=\mathrm{p}_{11}^{*} / \mathrm{p}_{\text {max }}^{*} \approx \mathrm{p}^{*} /(\sqrt{\mathrm{s}} / 2)$ where

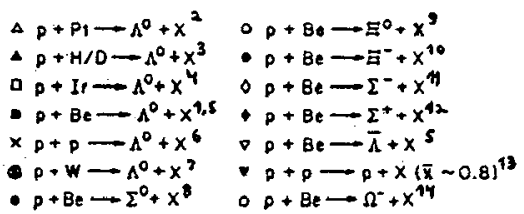
$\mathrm{p}^{*} 11$ is the hyperon momentum parallel to the projectile incident momentum in the center of mass of the mass of the interaction and $p_{\max }^{*}$ is the maximum value of that momentum. The magnitude of $x$ indicates the degree to which the hyperon is a fragment of the projectile if $x>0$ or the target if $x<0$.

Figure 1: Inclusive hyperon polarization from representative experiments using high energy proton projectiles. Single data points closest to $\mathrm{p}_{\mathrm{T}}=1 \mathrm{GeV} / \mathrm{c}, \mathrm{x}=0.5$ have been used so that the points do not, in most cases, reflect the total precision of each experiment. All polarizations are actually negative except for $\Sigma^{+}, \Sigma^{-}$and $\Sigma^{0}$ which are positive. The sign of the $\Omega^{-}$polarization has not been determined. The fraction of the parent particle momentum scattered perpendicular to the projectile incident momentum is $\mathrm{x}_{\mathrm{T}}=\mathrm{p}_{\mathrm{T}} / \mathrm{p}_{\max } \approx \mathrm{p}_{\mathrm{T}} /(\sqrt{\mathrm{s}} / 2)$ where $\mathrm{p}_{\mathrm{T}}$ is the transverse momentum of the hyperon. Since the strong interaction respects parity, the only allowed polarization is in the direction $\hat{\mathrm{n}}=\hat{\mathrm{p}}_{\text {in }} \mathrm{x}$ $\hat{\mathrm{p}}_{\mathrm{H}}$ where $\hat{\mathrm{p}}_{\mathrm{in}}$ is the direction of the parent particle and $\hat{p}_{\mathrm{H}}$ the direction of the hyperon. A11 inclusive hyperon polarizations measured at high energy are along this direction except for that initiated by a $\nu(\bar{v})$ beam.

In terms of the constituent quarks, proton fragmentation into a hyperon can be viewed as the replacement of one valence quark $\left(\Lambda, \Sigma^{+}, \Sigma^{\circ}\right)$, two valence quarks $\left(\Sigma^{-}, \Xi^{\circ}, \equiv^{-}\right)$or all three valence quarks $\left(\Omega^{-}, \bar{\Lambda}\right)$ in the original projectile. After the recombination of the new quarks into the baryon, the quark spin is usually deduced from the hyperon polarization using constituent quark wave functions. These static "SU(6)" wave functions have been remarkably successful, although not perfect, in predicting baryon magnetic moments. 15 Examination of the spin structure of these wave functions leads to the simple rule that the spin_of the hyperon is in the same direction as that of the strange quark for $\Lambda, \equiv, \Xi^{-}$and $\Omega$ and in the opposite direction for $\Sigma^{+}$, $\Sigma^{-}$and $\Sigma^{\circ}$. In all cases of inclusive high energy hyperon production by protons, the measured polarization of $\Lambda, \equiv^{\circ}$, and $\equiv^{-}$is in fact opposite that for 


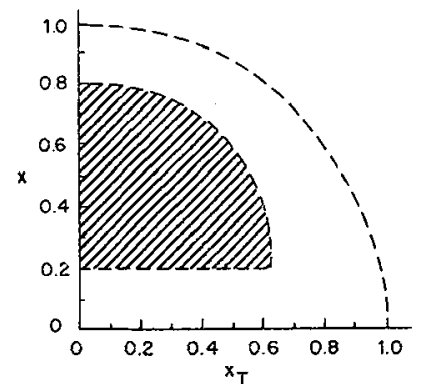

Figure 2: Kinematic region covered by inclusive $\Lambda$ polari-both $x$ and zation measurements. The data fall within the cross hatched region. The dashed line is the kinematic limit.

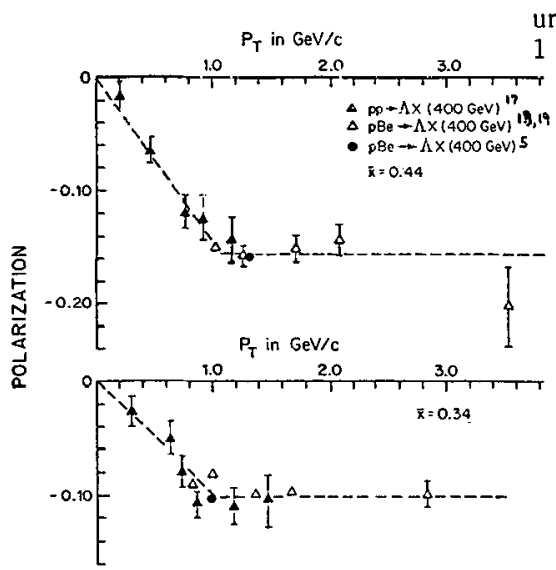

Figure 3: Inclusive $\Lambda$ polarization as a function of $\mathrm{p}_{\mathrm{T}}$ for approximate $1 y$ constant $x$. A sample of the data from three experiments all using the same spectrometer is shown. Errors when not shown are smaller than the points. The lines are drawn to guide the eye.

for $\mathrm{p}_{\mathrm{T}}>-\mathrm{GeV} / \mathrm{c}$ the polarization depends linearly on $x$ following what appears to be a universal curve as shown in Figure 4. Figure 5 gives a representation of this polarization as a function of $x$ and $\mathrm{p}_{\mathrm{T}}$. Because the polarization depends on both $\mathrm{x}$ and $\mathrm{P}_{\mathrm{T}}$, care must be taken in comparing data from different experiments. For $\mathrm{p}_{\mathrm{T}}>1 \mathrm{GeV} / \mathrm{c}$, data need only be compared as a function of $x$.

There ace two other factors which complicate the comparison of different experiments. The first is that ntil approximately pendent of $\mathrm{p}_{\mathrm{T}}$.

This behavior is illustrated in $17,19$.
$\Sigma^{+}, \Sigma^{-}$and $\Sigma^{\circ}$. 1-14 The $\Sigma^{\circ}$ and $\Omega^{-}$data, the magnitudes of which are shown in Figure 1, have large error bars and are still preliminary.

The most extensive investigation of the kinematic behavior of hyperon polarization has been carried out for $\Lambda$ production by protons. These data reach larger section range of 6 orders of magnitude. Most of the data, from $400 \mathrm{GeV}$ Fermilab experiments, investigate the kinematic region shown in Figure $2,0.2<x<0.8$, $0<\mathrm{p}_{\mathrm{T}}<3.7 \mathrm{GeV} / \mathrm{c}$. The $\Lambda$ polarization was found to

or a constant $x$, the polarization increases $\mathrm{GeV} / \mathrm{c}$. For $\mathrm{p}_{\mathrm{T}}>1$ $\mathrm{GeV} / \mathrm{c}$ the polarization is indeFigure 3. At low $\mathrm{P}_{\mathrm{T}}$ the polarization is only a very weak function of $x$ but

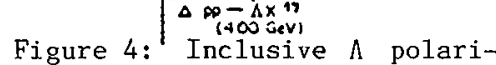
zation as a function of $x$ for a11 data with $\mathrm{p}_{\mathrm{T}}>1 \mathrm{GeV} / \mathrm{c}$ from two experiments. Also shown is low $\mathrm{p}_{\mathrm{T}}$ data. The line is drawn to guide the eye and will be used in the following figures to represent the polarization.

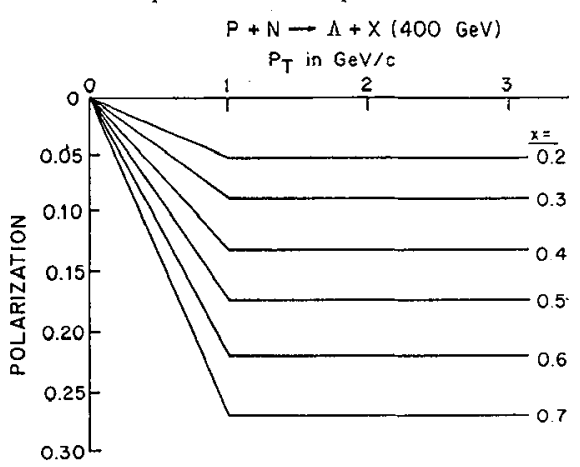

Figure 5: Schematic of the behavior of inclusive $\Lambda$ polarization as a function of $x$ and $p_{T}$. The data used to compile the picture comes from references 5 , 
experimenters tend to use different targets. The target dependence of $\Lambda$ polarization has been 9,19 and one at $28 \mathrm{GeV}^{3}$, and is shown in Figure 6. A11 experiments agree that for targets with larger atomic number the magnitude of the polarization decreases as might be expected from rescattering inside the nucleus. The effect is small, on the order of $3 \%$. No statistically significant target dependence has been observed for any other hyperon polarization. For simplicity it would be useful if future experiments would use the same target. Be has been used for the bulk of the existing data.

The second problem occurs only for $\Lambda$ production. The $\Lambda$ detected in the apparatus can come from either "direct" production or from $\Sigma^{O} \rightarrow \Lambda \gamma$ decay which occurs in the target. This $\Lambda$ from $\Sigma^{\circ}$ decay complicates the interpretation of the data since it carries - $\frac{1}{3}$ the polarization of the $\Sigma^{0}$ for complete $\Lambda$ acceptance. ${ }^{20}$ The $\Sigma^{0}$ contamination will always cause the measured $\Lambda$ polarization to be smaller in magnitude than the direct polarization. The direct $\Lambda$ polarization can be calculated if the amount of contamination and the $\Lambda$ polarization from $\Sigma^{\circ}$ decay is known $P($ measured $)=r(\Lambda) \frac{\mathrm{N}_{\Lambda}}{\mathrm{N}_{\Lambda}+\mathrm{N}_{\Sigma}}+P\left(\Lambda+\Sigma^{\circ}\right) \frac{\mathrm{N}_{\Sigma}}{\mathrm{N}_{\Lambda}+\mathrm{N}_{\Sigma}}$ where $P$ is the $\Lambda$ polarization as measured, from direct $\Lambda$ or from $\Sigma^{0} \rightarrow M$ decay as indicated, $\mathrm{N}_{\Lambda}$ is the number of direct $\Lambda^{\prime}$ 's detected, $N_{\Sigma}$ is the number of $\Lambda$ !s from $\Sigma^{0}$ decay detected, and $\mathrm{N}_{\Lambda}+\mathrm{N}_{\Sigma}$ is the total measured $\Lambda$ sample. A Brookhaven experiment measured the ratio $\mathrm{R}=\frac{\mathrm{N}_{\Sigma}}{\mathrm{N}_{\Lambda}+\mathrm{N}_{\Sigma}}$ at $28 \mathrm{GeV}$, see Figure 7 ,
by detecting the $\gamma$ as well as the $\Lambda$ and reconstructing the $\Sigma^{\circ}$ mass. 21 The result was that $R=28 \pm 6 \%$ for 28 $\mathrm{GeV}$ protons incident on a Be target and was approximately constant for $.35<\mathrm{x}<.80,0.6 \mathrm{GeV} / \mathrm{c}<\mathrm{p}_{\mathrm{T}}<1.3 \mathrm{GeV} / \mathrm{c}$. Since the difference between the $\Lambda$ and $\Sigma^{\circ}$ depends only on quark spins, this result should follow from any model which predicts hyperon polarzation. Of the few such models which exist, none give the correct result. ${ }^{22}$ Using the measured value of $R$,

$$
P(\Lambda)=(1.4)\left[P(\text { measured })+\left(\frac{1}{10}\right) P\left(\Sigma^{0}\right)\right]
$$


where $P\left(\Sigma^{0}\right)$ is the polarization of the produced ${ }^{10} \Sigma^{0}$ s. If $\mathrm{P}\left(\Sigma^{0}\right)$ has approximately the same magnitude as $P$ (measured), its actual value is not important in determining the correction. In fact this same experiment has a preliminary determination of $\mathrm{P}\left(\Sigma^{0}\right)=+0.28 \pm 0.09$ which is approximately equal to -P(measured). ${ }^{8}$ Thus all $\wedge$ polarization measurements at $28 \mathrm{GeV}$ should be multiplied by approximately 1.4 . Although this correction is not known for any other energy, it is not unreasonable to assume its validity for higher energies. However, in the following comparisons between $\Lambda$ polarization at different energies and between $\wedge$ polarization and that of other hyperons, no correction has been made for $\Sigma^{0}$ contamination. In

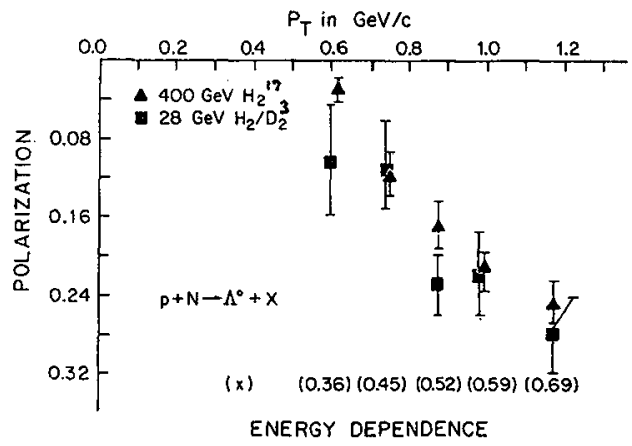

Figure 8: Comparison of inclusive polarization produced by 28 and $400 \mathrm{GeV}$ protons. The $400 \mathrm{GeV}$ data has been interpolated when necessary, so that it matches the $x$ value of the $28 \mathrm{GeV}$ data at each $\mathrm{p}_{\mathrm{T}}$.

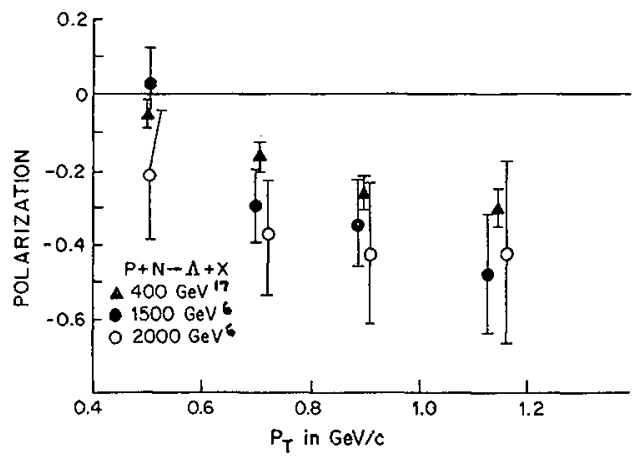

Figure 9: Comparison of inclusive polarization produced by 400 protons with $26 / 26$ and $31 / 31 \mathrm{GeV}$ proton-proton collisions. The $400 \mathrm{GeV}$ data has been interpolated to match the estimated $\mathrm{x}$ value of the collider data at each $p_{T}$.

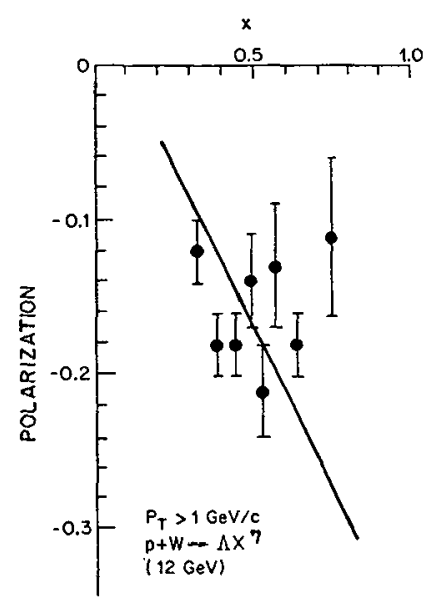

most cases the error bars are so large that a correction of 1.4 would not affect the qualitative conclusions.

The polarization of $\Lambda$ 's produced by protons has been measured at proton energies
of from $12 \mathrm{GeV}$ at KEK ${ }^{7}$ to the equivalent of $2000 \mathrm{GeV}$ at the ISR. ${ }^{6,23}$ Given the problems of comparing one experiment to another there is remarkable agreement in both the magnitude of the polarization and its kinematic behavior. Comparisons are shown in Figures 8 and 9. In both cases the $400 \mathrm{GeV}$ data has been interpolated when necessary using the kinematic behavior shown in Figure 5 so that the comparison is always made at approximately the same $\mathrm{x}$ for a given $\mathrm{P}_{\mathrm{T}}$. Since the $\mathrm{x}$ values for the ISR data are not published, I have estimated them using the acceptance of the experiment. ${ }^{24}$ In Figure 10 the $12 \mathrm{GeV}$ data with a $\mathrm{p}_{\mathrm{T}}$ above $0.9 \mathrm{GeV} / \mathrm{c}$ is compared to a line representing the behavior of the $400 \mathrm{GeV}$ data as a function of $\mathrm{x}$. 


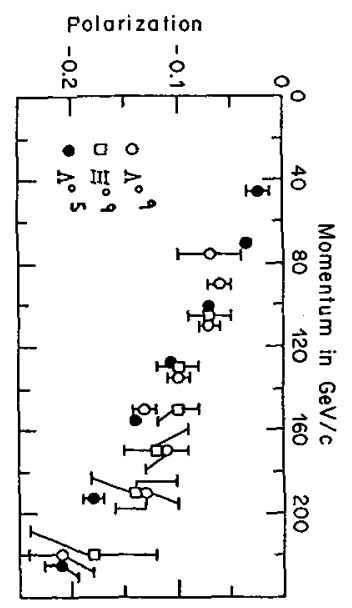

Figure 11: Inclusive $\equiv^{\circ}$ and $\Lambda$ polarization produced by $400 \mathrm{GeV}$ protons. Open points are data from the same experiment. Solid points are from an earlier experiment. A11 data were taken at a production angle of $7.2 \mathrm{mrad}$.

The polarizations of other hyperons produced by $400 \mathrm{GeV}$ protons have been measured and show a remarkable similarity. Figure 11 shows a comparison of $\Lambda$ and $\equiv$ polarization taken in the same experiment at the same time. Also shown is the $\Lambda$ polarization measured in an earlier experiment with essentially the same apparatus at the same production angle using the same target. The agreement between the $\Lambda$ and $\equiv$ polarization is as good as the agreement between the two sets of $\Lambda$ data. Figure 12 shows the $\equiv$ data for $\mathrm{P}_{\mathrm{T}}>0.9 \mathrm{GeV} / \mathrm{c}$ as a function of $x$ compared to the line representing $\Lambda$ polarization. Figure 13 shows $\equiv-$ data in the same way. ${ }^{10}$ The ${ }^{-}$polarization may be slightly smaller than that of $\Lambda$ and $\equiv$

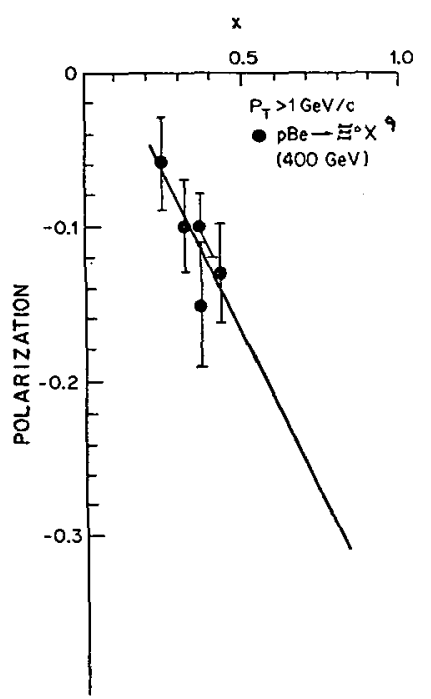

Figure 12: Inclusive ${ }^{\circ}$ polarization produced by $400 \mathrm{GeV}$ protons. The line represents the 400 $\mathrm{GeV} \wedge$ data from Figure 4.

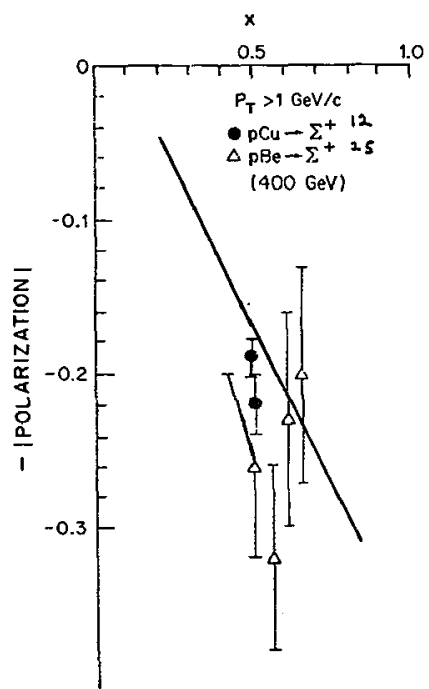

Figure 14: Inclusive $\boldsymbol{\Sigma}^{+}$ polarization from $400 \mathrm{GeV}$ protons. The polarization is multiplied by -1 . The line represents the 400 $\mathrm{GeV} \wedge$ data from Figure 4.

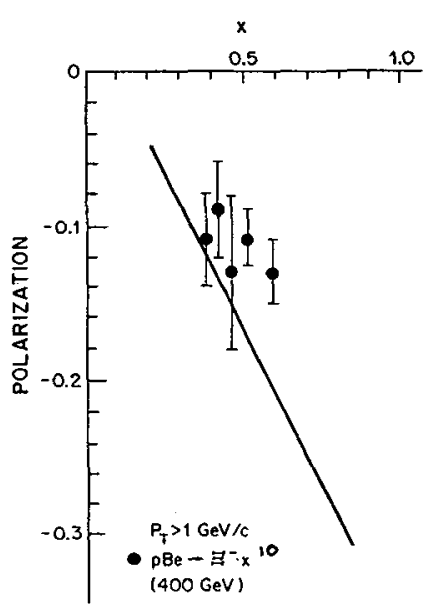

Figure 13: Inclusive polarization produced by $400 \mathrm{GeV}$ protons. The line represents the $400 \mathrm{GeV}$ data from Figure 4.

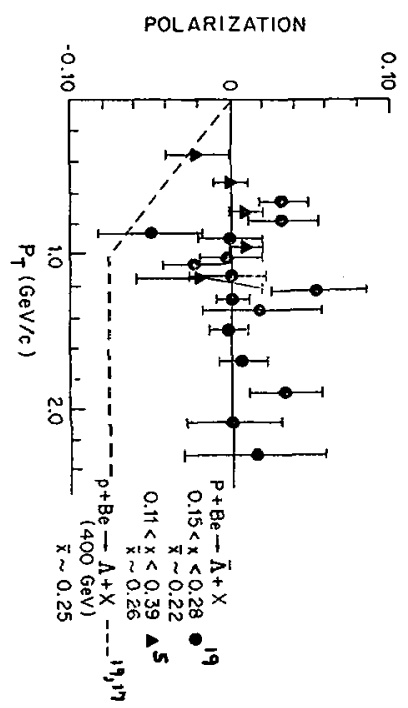

Figure 15: Inclusive $\pi$ polarization from $400 \mathrm{GeV}$ protons. The dashed line represents the $\Lambda$ polarization for the same kinematics. 
but the overall behavior appears similar. Figure 14 shows the $\Sigma^{+}$polarization multiplied by $-1 .{ }^{12,25}$ Again there is good agreement. Finally Figure 15 gives the $\bar{\Lambda}$ polarization as a function of $\mathrm{P}_{\mathrm{T}} \cdot 5,18,19$ Also shown is a line representing the polarization at the same average value of $x$. It is clear that $\bar{\Lambda}$ polarization is much smaller than the $\Lambda$ polarization is consistent with zero out to a $\mathrm{p}_{\mathrm{T}}$ of $2 \mathrm{GeV} / \mathrm{c}$.

The agreement of all the hyperon polarization data in the limited kinematic regions measured leads one to believe that a single, simple mechanism is responsible for all hyperon production. One should remember, however, that $\Sigma^{-}, \Sigma^{\circ}$ and $\Omega^{-}$are only measured at one point and with larger errors. Also, no correction has been made to the $\Lambda$ polarization for $\Sigma^{0}$ dilution. A different mechanism must be responsible for antihyperon production since $\bar{\Lambda}$ is not polarized.

The situation for $\Lambda$ polarization produced by particles other than protons is not so clear. A Brookhaven experiment has published $\Lambda$ polarization produced by a $16 \mathrm{GeV} \pi^{-}$beam. ${ }^{26}$ Figure 16 shows this data together with the curve representing

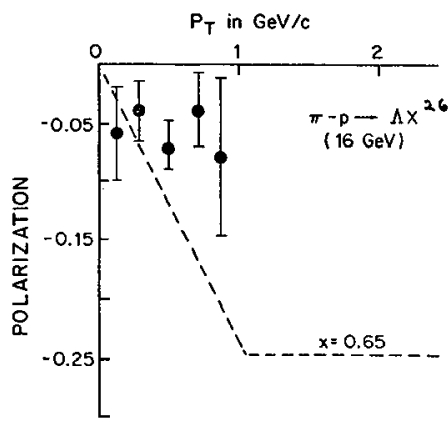

Figure 16: Inclusive polarization from $16 \mathrm{GeV}$ pions. The dashed line represents the $400 \mathrm{GeV}$ polarization for the same kinematics from Figure 5.

proton production but in this case the strange quark is originally. unpolarized since it comes from the $K^{2}$. The polarization as a function of $\mathrm{p}_{\mathrm{T}}$

appears to be decreasing with energy which could mean that it is unrelated to the high energy proton produced polarization. However, since the value of $x$ for each point is not given and probably decreases with beam energy the situation is not clear.

$\Lambda$ polarization from protons at the same average value of $x$. It is clear these are different. The experimenters state that the polarization measurement may suffer from biases which they could not eliminate. The data for $\Lambda$ polarization from a $\mathrm{K}^{-}$beam has been reviewed at this conference by T. Haupt. Most of this data is from low statistics bubble chamber exposures dominated by energies below $10 \mathrm{GeV} .{ }^{27}$ At low energy large polarizations are very common due to the domination of a few exclusive channels. Figure 17 shows $\Lambda$ polarization as a function of $p_{\mathrm{T}}$ for $\mathrm{x}<0$, in effect protons producing $\Lambda^{\prime}$ s on a $K^{-}$target. ${ }^{28}$ The data have large errors and the value of $x$ at each point has not been given so it is hard to compare with the proton beam data. The sign of the polarization is negative as expected. Figure 18a gives the polarization of $\Lambda^{\prime}$ s produced by $\mathrm{K}^{-}, \mathrm{x}>0$. Again, the sign of the polarization is the same as for
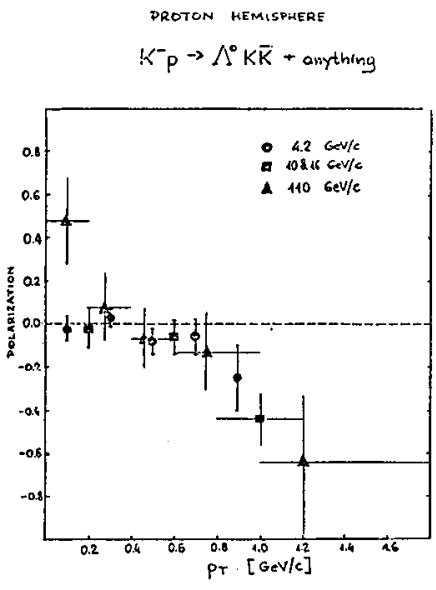

Figure 17: Inc1usive polarization from proton fragmentation using a $\mathrm{K}^{-}$ beam. From the presentation of T. Haupt.

\section{KAON HENISPHERE}
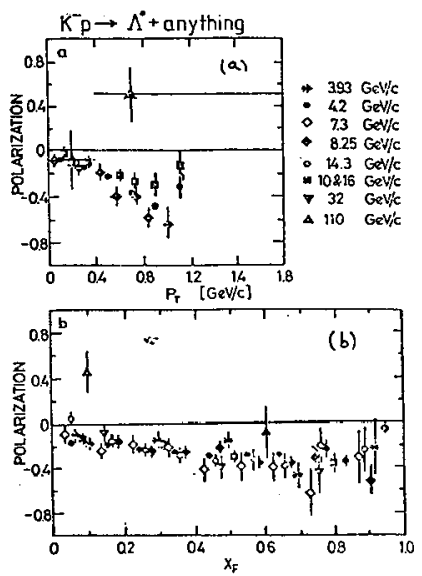

Figure 18: Inclusive polarization from $\mathrm{K}^{-}$fragmentation as a function of (a) $p_{T}$, (b) $x$. From the presentation of $\mathrm{T}$. Haupt. 
Figure 18b shows the same data as a function of $\mathrm{x}$ including some from additional experiments. 28 There is no evident energy dependence but the value of $p_{T}$ for each point, which dominates the behavior of proton produced polarization at low $\mathrm{p}_{\mathrm{T}}$, is not given. A high statistics, high energy measurement of $\Lambda$ polarization from $\mathrm{K}^{-}$production would be very helpful. This reaction can only give polarized strange quarks by a recombination as opposed to a quark production mechanism since the produced (ud) diquark carries no spin information in the $\Lambda$.

Finally there is some preliminary data from BEBC using neutrinos to produce $\Lambda^{\prime}$ s. $^{29}$ For $x<0, v(\bar{v})+p \rightarrow \Lambda+\mu+$ anything is equivalent to the charged current reaction $\mathrm{p}+\mathrm{W} \rightarrow \mathrm{A}+$ anything. The spin dependence of the interaction of the $W$ with the proton is certain1y better understood than the strong interaction. With only a few hundred events which have $Q^{2}<4(\mathrm{GeV} / \mathrm{c})^{2}$, the $\Lambda$ polarization observed in the production plane was $0.63 \pm 0.22$ for the $\nu$ beam and $0.50 \pm 0.20$ for the $\bar{v}$ beam. Perpendicular to the production plane the polarization was consistent with zero, $0.05 \pm 0.22(v)$ and $-0.02 \pm 0.20(\bar{v})$. The existence of $\Lambda$ polarization in the production plane is expected for a charged current producing $\Lambda^{\prime}$ s via $Y^{*}$ resonances. Hopefully, future large neutrino experiments will have more data of this type with better statistics.

In summary, high energy inclusive polarization is a large and common phenomenon. It occurs in almost every reaction investigated. I believe that the following picture is emerging from the data of high energy hyperons produced by protons. Without a compelling theoretical guide, this picture is based on the data presented in this review filtered by my own conservative prejudices and desire for simplicity.

1. A11 hyperons produced by proton fragmentation are polarized perpendicular to the production plane. Antihyperons are not.

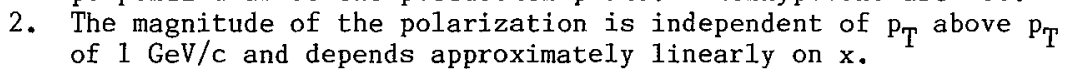

3. The magnitude of the polarization depends approximately linearly on $\mathrm{p}_{\mathrm{T}}$ below $\mathrm{p}_{\mathrm{T}}$ of $0.8 \mathrm{GeV} / \mathrm{c}$.

4. The magnitude and kinematic behavior of the polarization is remarkably energy independent for projectiles above $12 \mathrm{GeV}$. If anything it increases with energy.

5. The direction of polarization of the strange quarks is the same for all hyperons. The opposite sign of the $\Sigma$ polarizations arises naturally from using the static quark wavefunctions in a straight forward way.

6. The $\Sigma^{3}$ to $\Lambda$ ratio at production is approximately $40 \%$. This ratio does not arise naturally from using the static quark wavefunctions in a straight forward way. Increasing the $\Lambda$ polarization by a factor of 1.4 to correct for $\Sigma^{O}$ dilution means the $\equiv$ polarizations are smaller than those of the $\Lambda$.

7. Nuclear targets reduce the polarization of the hyperons which emerge. Rescattering in the nucleus probab1y reduces the correlation between scattering, momentum, and spin direction.

At this time there is no satisfactory model which shows how this behavior fits into the standard theory of strong interactions. Additional data could help clarify the situation. The most useful measurements would be large statistics experiments at high energy with good coverage in $x$ and $\mathrm{p}_{\mathrm{T}}$. The following is a list of examples.

The $\Lambda$ polarization at higher $\mathrm{p}_{\mathrm{T}}$ to further press $\mathrm{QCD} .{ }^{30}$

The $\Sigma^{\%} / \Lambda$ ratio at $400 \mathrm{GeV}$ or above to see if the $28 \mathrm{GeV}$ results apply to higher energy.

The $\Lambda$ and $\bar{\Lambda}$ polarization at the CERN or Tevatron collider to test the energy dependence.

A detailed $x, p_{T}$ map of the polarization of hyperons other than $\Lambda$. In the case of the $\Omega$ any additional information would be useful. 
The contribution to the $\Lambda$ polarization from each exclusive channel. Data using beams other than protons. The situation for $\mathrm{K}^{-} \rightarrow \Lambda$ needs to be clarified.

A11 of the above experiments are long and difficult. There is always the possibility of a surprise which will change the picture of hyperon polarization. The most likely result, however, would be to fill out the existing picture which remains a puzzle for theory. From this point of view, the greatest need at this time is further theoretical work.

\section{References}

1. G. Bunce, et a1, PRL 36, 1113 (1976).

2. K. Heller et a1., PL $\underline{68 \mathrm{~B}}, 480$ (1977).

3. K. Raychandhuri et a1., PL 90B, 319 (1980).

4. F. Lomanno et a1., PRL 43, 1905 (1979).

5. K. Heller et al., PRL 41, 607 (1978).

6. S. Erhan et aI, PL 82B, 1325 (1979).

7. F. Abe et al, PRL 50, 1102 (1983).

8. C. Dukes, Univ. of Mich. Thesis (1985), unpublished.

9. K. Heller et al, PRL 51, 2025 (1983).

10. R. Rameika, Rutgers Univ. Thesis (1981), unpublished.

11. L. Deck et a1, PR D28, 1 (1983).

12. C. Ankenbrandt et al, PRL 51, 863 (1983).

13. R. Polvado et a1, PRL 42, 1325 (1979).

14. B. Luk, Rutgers Univ. Thesis (1983), unpublished.

15. For a recent review, H. Lipkin, NP B241, 477 (1984) and the references therein.

16. K. Heller, High Energy Physics - 1980, ed. L. Durand and L. Pondrom, AIP Conf. Proc. 68, 61 (1981).

17. R. Grobel, Univ. of Wisc. Thesis (1980), unpublished.

18. B. Lundberg et a1, High Energy Spin Physics - 1982 ed. G. Bunce, AIP Conf. Proc. 95, 83 (1983).

19. B. Lundberg, Univ. of Wisc. Thesis (1984), unpublished.

20. R. Gatto, Phys. Rev. 109, 610 (1958).

21. M. Su1livan et a1., PL 142B, 451 (1984).

22. B. Anderson, G. Gustafson, and G. Ingelman, PL 85B, 417 (1979);

T. DeGrand and H. Miettinen, PR D24, 2419 (1981); J. Szwed, PL 105B, 403 (1981).

23. P. Schlein, this conference.

24. J. Rander, UCLA Thesis (1976), unpublished.

25. C. Wilkinson et al., PRL 46,803 (1981).

26. J. Bensinger et al. . PRL $\overline{50}, 313$ (1983).

27. A. Borg et al., NC 22A, 559 (1974); S. Ganguli et al., NC 44A, 345 (1978); S. Chung et al., PR D11, 1010 (1975); M. Baubillier et al., NP B148, 18 (1979); J. Bartsch et ai., NP B40, 103 (1972); H. Grassler et al., NP B136, 386 (1978); H. Abramowicz et a1., NP B105, 222 (1976), M. Faccini-Turluer et al., ZP C1, 19 (1979); U. Gensch et a1., NP B173, 154 (1980).

28. T. Haupt, this conference.

29. H. Grassler et a1., MPI Munich, Preprint (1984).

30. G. Kane, J. Pumplin and W. Repko, Phys. Rev. Lett. 41, 1257 (1978). 